January 1992

UMDGR-91-134

\title{
REMARKS ON PURE SPIN CONNECTION FORMULATIONS OF GRAVITY
}

\author{
Riccardo Capovilla ${ }^{(1)}$ and Ted Jacobson ${ }^{(2)}$ \\ (1) Centro de Investigaciones y Estudios Avanzados, I.P.N. \\ Apdo. Postal 14-740 - Mexico 14, D.F. - Mexico \\ (2) Department of Physics and Astronomy \\ University of Maryland, College Park, MD 20742
}

\begin{abstract}
In the derivation of a pure spin connection action functional for gravity two methods have been proposed. The first starts from a first order lagrangian formulation, the second from a hamiltonian formulation. In this note we show that they lead to identical results.
\end{abstract}

PACS: 04.20.Cv, 04.20.Fy, 02.40.+m 
As shown by Ashtekar, there is a hamiltonian formulation of general relativity which uses the (spatial) self-dual spin connection as the configuration space variable. [1] The 3-metric is essentially the conjugate momentum, and therefore loses some of its priviledged status in the theory. It is possible to go one step further, and give a covariant lagrangian formulation of general relativity which employs only the (space-time) self-dual spin connection and a scalar density as the gravitational variables. 22, 31 In this "pure spin connection formulation", the spacetime metric is merely a derived quantity, and general relativity appears as a generally covariant gauge theory of an SL(2,C) connection.

For a spacetime with a metric of lorentzian signature, the use of the self-dual spin connection as a field variable implies the use of complex variables. Properly speaking therefore the pure spin connection formulation describes complex general relativity, and reality conditions need to be imposed a posteriori. For the sake of simplicity, in the following we will consider only the complex case. The appropriate reality conditions are discussed in detail in Ref. [3].

The pure spin connection formalism was originally derived starting from a first order formulation of general relativity, and solving the variational equations in favor of the self-dual spin connection, an undetermined scalar density, and the matter fields. [2, 3] It was later shown that one can also arrive there directly from Ashtekar's hamiltonian formalism, by performing an inverse Legendre transform. [4, 5, 6] In the vacuum case the pure spin connection action turns out to be polynomial in the basic field variables. For massless scalar and spinor field couplings, it remains polynomial. However in the case of a non-vanishing cosmological constant, or for massive scalar or gauge field couplings, it becomes non-polynomial and rather complicated.

A caveat which needs to be mentioned is that the equivalence between the usual formulation of general relativity and its pure spin connection version holds only modulo a certain non-degeneracy condition. The form of this condition depends on the type of matter coupling considered, and on the presence of a cosmological constant. In the vacuum case, for a vanishing cosmological constant, the condition is that the self-dual part of the Weyl tensor, thought as three by three matrix, must be non-degenerate. This implies a restriction on the class of spacetimes for which the equivalence holds. In particular, it fails for spacetimes of type $\{3,1\},\{4\}$ in the algebraic classification of the Weyl tensor. [7]

A discrepancy appeared in the non-polynomial cases between the results obtained by the two approaches mentioned above. It turns out that in these cases 
there was an error in the derivation of a pure spin connection action functional of [3]. 80 In this note we shall show that in fact the two approaches lead to identical results.

In order to demonstrate the equivalence we have found it useful to slightly rewrite the first order formulation of general relativity that serves as the starting point in the derivation of a pure spin-connection action. In particular, we shall write the constraint on the trace of the Lagrange multiplier matrix field $X$ (which in the vacuum case turns out to correspond to the self-dual part of the Weyl tensor) in a form alternative to the obvious choice $\operatorname{Tr} X=\Lambda$ used in [3]. Dadhich et al. have previously pointed out that different ways of imposing this constraint may lead to different pure spin-connection actions. [9] Such differing actions are related by field redefinition of the scalar density that appears in the final form. In the polynomial cases, the result turns out to be identical to what was obtained before.

We now go on to the calculations. First we rederive the pure spin-connection action in the vacuum case. This rederivation turns out to be simpler than the original derivation given in [3], because an awkward step requiring the extraction of the square root of a $3 \times 3$ matrix is eliminated. In fact it is in this step that the error was made in the cosmological constant case. [8]

Complex vacuum general relativity can be described by the first order action functional introduced originally by Plebanski [10] (see also [11], [12]). This action is a functional of a trio of 2 -forms $\Sigma^{i}$, an $\mathrm{SO}(3, \mathrm{C})$ connection 1-form $\omega^{i}$, with curvature $R_{i}:=d \omega_{i}+\frac{1}{2} \epsilon_{i j k} \omega^{j} \wedge \omega^{k}$, and a symmetric and traceless Lagrange multiplier field $\Psi_{i j}$ 円.

The action can be written in the form

$$
S\left[\Sigma^{i}, \omega_{i}, \Psi_{i j}, \mu\right]=\int\left[\Sigma^{i} \wedge R_{i}-\frac{1}{2} \Psi_{i j} \Sigma^{i} \wedge \Sigma^{j}+\mu \Psi_{i}^{i}\right]
$$

Let us briefly discuss the variational equations for this action. (For a more detailed discussion see e.g. [12.) The Lagrange multiplier $\mu$ enforces explicitly the condition that $\Psi_{i j}$ is traceless. In turn, the $\Psi_{i j}$ equations of motion imply that the trio of 2forms $\Sigma^{i}$ are subject to the constraint $\Sigma^{i} \wedge \Sigma^{j} \propto \delta^{i j}$. The content of this constraint is that there exists a tetrad of 1 -forms $\theta^{a}$ such that the trio of 2 -forms corresponds to the self-dual part of $\theta^{a} \wedge \theta^{b}$. The trio of 2-forms $\Sigma^{i}$ play the role of basic "metric variables". The $\omega_{i}$ field equation implies then that $\omega_{i}$ itself is the self-dual part

\footnotetext{
${ }^{1}$ Lower case latin letters from the middle of the alphabet denote $\mathrm{SO}(3, \mathrm{C})$ indices, which are raised and lowered with the Kronecker delta $\delta^{i j}$.
} 
of the spin connection compatible with the tetrad $\theta^{a}$. Finally, the $\Sigma^{i}$ equation of motion, $R_{i}=\Psi_{i j} \Sigma^{j}$, says that the curvature is pure Weyl, i.e. that the spacetime metric $g_{\mu \nu}=\theta_{\mu}{ }^{a} \theta_{\nu}{ }^{b} \eta_{a b}$ is Ricci flat. The metric density can also be expressed directly in terms of the 2 -forms $\Sigma^{i}$ as

$$
\sqrt{g} g_{\mu \nu}=\frac{1}{3} \Sigma_{\mu \alpha}^{i} \Sigma_{\beta \gamma}^{j} \sum_{\delta \nu}^{k} \epsilon^{\alpha \beta \gamma \delta} \epsilon_{i j k}
$$

In this sense one can say that $\Sigma^{i}$ is a "cube root" of the metric.

Starting from the action (1), it is possible to eliminate both $\Sigma^{i}$ and $\Psi_{i j}$, solving for them in terms of the curvature 2 -form $R_{i}$ and an undetermined scalar density by use of the variational equations [2, 3]. This results in a pure spin connection formulation of (complex) vacuum general relativity given by

$$
S[\omega, \eta]=\int \eta\left[\operatorname{Tr}\left(M^{2}\right)-\frac{1}{2}(\operatorname{Tr} M)^{2}\right]
$$

where we have defined the matrix (of weight +1 )

$$
M_{i j}:=R_{i} \wedge R_{j}
$$

and $\eta$ is a scalar density of weight -1 . In terms of $\eta$ and $\omega_{i}$, the spacetime metric is given by

$$
g_{\mu \nu}=\frac{1}{3} \eta R_{\mu \alpha}^{i} R_{\beta \gamma}^{j} R_{\delta \nu}^{k} \epsilon^{\alpha \beta \gamma \delta} \epsilon_{i j k}
$$

From the point of view of the pure connection formulation, the way to identify the metric is to express the $\omega_{i}$ equation of motion as $D \Sigma^{i}=0$ for some 2 -forms $\Sigma^{i}$. These $\Sigma^{i}$ are then the cube root of the metric density. They are determined up to a constant multiplicative factor.

The equivalence between the actions (1) and (2) holds if and only if $\Psi_{i j}$ is invertible. The reason is that this assumption is necessary in order to solve the $\Sigma$ variational equation, $R_{i}=\Psi_{i j} \Sigma^{j}$, for $\Sigma^{i}$ itself, i.e. $\Sigma^{i}=\left(\Psi^{-1}\right)^{i j} R_{j}$. This implies the restriction mentioned previously on the class of spacetimes for which the pure spin-connection formulation is equivalent to general relativity.

We shall now derive the pure spin-connection action (2), and its generalization with a cosmological constant, from the first order action (四). In preparation, the constraint on $\operatorname{Tr} \Psi$ will first be rewritten. This is done with the help of the characteristic equation satisfied by any $3 \times 3$ matrix $A$, i.e.

$$
A^{3}-(\operatorname{Tr} A) A^{2}+\frac{1}{2}\left((\operatorname{Tr} A)^{2}-\operatorname{Tr} A^{2}\right) A-\operatorname{det} A=0
$$


When $\Psi$ is non degenerate, which we need to assume anyway, we can take $A=\Psi^{-1}$, multiply through by $\Psi$, and take the trace to obtain

$$
\operatorname{Tr} \Psi=\frac{1}{2} \operatorname{det} \Psi\left[\left(\operatorname{Tr} \Psi^{-1}\right)^{2}-\operatorname{Tr} \Psi^{-2}\right]
$$

With this alternative expression for $\operatorname{Tr} \Psi$, the action (11) takes the form

$$
S[\Sigma, \omega, \Psi, \rho]=\int \operatorname{Tr}\left[\Sigma \wedge R-\frac{1}{2} \Psi \Sigma \wedge \Sigma\right]+\rho\left[\left(\operatorname{Tr} \Psi^{-1}\right)^{2}-\operatorname{Tr} \Psi^{-2}\right]
$$

where the Lagrange multiplier $\mu$ has been traded in for $\rho$, which is related to $\mu$ by $\rho=\mu(\operatorname{det} \Psi) / 2$. (The indices have been suppressed in a convenient "matrix" notation.)

The $\Sigma$ variational equation is $R=\Psi \Sigma$, which is solved by $\Sigma=\Psi^{-1} R$, provided that $\Psi$ is invertible. Since one has solved for the same variable $\Sigma$ that was varied in the action, the solution can be substituted back into the action, yielding

$$
S[\omega, \Psi, \rho]=\int \frac{1}{2} \operatorname{Tr}\left[\mathrm{J}^{-1} M\right]+\rho\left[\left(\operatorname{Tr} \Psi^{-1}\right)^{2}-\operatorname{Tr} \Psi^{-2}\right]
$$

where $M$ is defined as in (3). At this point one wants to solve for $\Psi$ from the $\Psi$ equation of motion, and here the alternative form of the trace constraint turns out to be more convenient. The variation of (7) with respect to $\Psi^{-1}$ gives

$$
M=4 \rho\left[\Psi^{-1}-\left(\operatorname{Tr} \Psi^{-1}\right) I\right]
$$

which is easily solved for $\Psi^{-1}$ with respect to $M$ and $\rho$, i.e.

$$
\Psi^{-1}=(4 \rho)^{-1}\left[M-\frac{1}{2}(\operatorname{Tr} M) I\right]
$$

When this solution is substituted in the action (7), one recovers the action (2), with $\eta=(16 \rho)^{-1}$.

One may wonder what happens if in the constraint term in the action (6), the relative coefficient -1 is replaced by an arbitrary parameter. A derivation which follows the footsteps of the one just given for vacuum general relativity shows that the elimination of $\Sigma$ and $\Psi$ recovers the family of generally covariant gauge theories discussed in [13] which generalize the pure spin connection action for vacuum general relativity (see also [14]).

We now turn to the case of a non-vanishing cosmological constant. The addition of a cosmological constant term to (1) yields the action

$$
S[\Sigma, \omega, X, \mu]=\int\left\{\operatorname{Tr}\left[\Sigma \wedge R-\frac{1}{2} X \Sigma \wedge \Sigma\right]+\mu(\operatorname{Tr} X-\mathrm{€})\right\}
$$


where we have defined $X:=\Psi+(1 / 3) \Lambda I$, and $\Lambda$ is the cosmological constant. The Lagrange multiplier $\mu$ enforces the constraint that $X$ has trace equal to $\Lambda$, or, in other words, that $\Psi$ is traceless.

Provided that the assumption of invertibility of $\mathrm{X}$ holds, (5) can be used to replace the constraint $\operatorname{Tr} X-\Lambda=0$ in the action (8) by the equivalent constraint

$$
\left(\operatorname{Tr} X^{-1}\right)^{2}-\left(\operatorname{Tr} X^{-2}\right)-2(\operatorname{det} X)^{-1} \mathrm{E}=0
$$

This yields the action

$$
\int\left\{\operatorname{Tr}\left[\Sigma \wedge R-\frac{1}{2} X \Sigma \wedge \Sigma\right]+\rho\left[\left(\operatorname{Tr} \mathrm{X}^{-1}\right)^{2}-\left(\operatorname{Tr} X^{-2}\right)-2(\operatorname{det} X)^{-1} \Lambda\right]\right\}
$$

where now $\mu$ has been traded for $\rho=\mu(\operatorname{det} X) / 2$.

Solving the $\Sigma$ equation of motion for $\Sigma$ itself gives

$$
\int\left\{\frac{1}{2} \operatorname{Tr}\left(X^{-1} M\right)+\rho\left[\left(\operatorname{Tr} \mathrm{X}^{-1}\right)^{2}-\left(\operatorname{Tr} X^{-2}\right)-2(\operatorname{det} X)^{-1} \Lambda\right]\right\}
$$

where $M$ is defined as in (3). Now varying this action (11) with respect to $X^{-1}$ gives

$$
M=4 \rho\left[X^{-1}-\left(\operatorname{Tr} X^{-1}\right) \mathbf{I}+\Lambda(\operatorname{det} X)^{-1} X\right]
$$

which is a matrix equation quadratic in $X^{-1}$. (That the last term is quadratic in $X^{-1}$ follows from the characteristic equation (4) with $A=X^{-1}$.) If this equation could be solved for $X$ in terms of $M$ and $\rho$, the solution $X(M, \rho)$ could then be substituted back into the action (11), yielding an action involving only the spin connection and $\rho$.

Now in fact it does not seem to be possible to obtain a solution for $X(M, \rho)$ in closed form. Moreover, such a solution may be non-unique or may not exist at all for certain values of $(M, \rho)$. (For instance, the simpler equation $M=B^{2}$ has many solutions for $B$ if $M=I$ and none if all elements of $M$ vanish except for $M_{12}=M_{33}=1$.)

Peldán also faces the problem of solving the matrix equation (12) in his alternative approach. [6] His method of dealing with this situation is the following. One supposes there is a unique solution $Y=Y(M, \rho):=X^{-1}(M, \rho)$ to (12). One then makes the move of substituting the expression (12) for $M$ into the action (8), obtaining

$$
\int \rho\left[\operatorname{Tr} Y^{2}-(\operatorname{Tr} Y)^{2}+4 \Lambda(\operatorname{det} Y)\right]
$$

This action involves only the particular combination of invariants

$$
z=z\left(\operatorname{Tr} M, \operatorname{Tr} M^{2}, \operatorname{det} M, \rho\right):=\operatorname{Tr} Y^{2}-(\operatorname{Tr} Y)^{2}+4 \Lambda(\operatorname{det} Y)
$$


Although one cannot solve (12) explicitly for $Y=X^{-1}$, it turns out that one can solve for the invariant $z$. Is this sufficient? It can happen that the matrix equation (12) has no solution but that there is a solution for its invariants. Ignoring these pathological cases, to solve for $z$, consider the independent invariants of the equation (12),

$$
\begin{aligned}
\operatorname{Tr} M & =2 \rho\left\{\Lambda\left[(\operatorname{Tr} Y)^{2}-\left(\operatorname{Tr} Y^{2}\right)\right]-4(\operatorname{Tr} Y)\right\} \\
\operatorname{Tr} M^{2} & =16 \rho^{2}\left\{\left(\operatorname{Tr} Y^{2}\right)+(\operatorname{Tr} Y)^{2}-\Lambda(\operatorname{Tr} Y)\left[(\operatorname{Tr} Y)^{2}-\left(\operatorname{Tr} Y^{2}\right)\right]+6 \Lambda(\operatorname{det} Y)\right. \\
& \left.-2 \Lambda^{2}(\operatorname{Tr} Y)(\operatorname{det} Y)+\frac{1}{4} \Lambda^{2}\left[(\operatorname{Tr} Y)^{2}-\left(\operatorname{Tr} Y^{2}\right)\right]^{2}\right\} \\
\operatorname{det} M & =64 \rho^{3}\left\{(\operatorname{det} Y)-\frac{1}{2}(\operatorname{Tr} Y)\left[(\operatorname{Tr} Y)^{2}-\left(\operatorname{Tr} Y^{2}\right)\right]+\frac{1}{4} \Lambda\left[(\operatorname{Tr} Y)^{2}-\left(\operatorname{Tr} Y^{2}\right)\right]^{2}\right. \\
& \left.+\Lambda(\operatorname{Tr} Y)(\operatorname{det} Y)-\Lambda^{2}(\operatorname{det} Y)\left[(\operatorname{Tr} Y)^{2}-\left(\operatorname{Tr} Y^{2}\right)\right]+\Lambda^{3}(\operatorname{det} Y)^{2}\right\}
\end{aligned}
$$

(The last expression is obtained by computing $\operatorname{Tr} M^{3}$ and then using the characteristic equation (4) for $M$.) Although we have not been able to solve these equations for $\operatorname{Tr} Y, \operatorname{Tr} Y^{2}$ and $\operatorname{det} Y$ independently, one can in fact solve for the combination $z$. It turns out that it obeys the quadratic equation

$$
\frac{\Lambda^{2}}{8} z^{2}-\left(1+\frac{\Lambda}{8 \rho} \operatorname{Tr} M\right) z+\frac{1}{16 \rho^{2}}\left[\operatorname{Tr} M^{2}-\frac{1}{2}(\operatorname{Tr} M)^{2}\right]-\frac{\Lambda}{32 \rho^{3}} \operatorname{det} M=0
$$

The integrand in (13) can then be written in terms of $M$ and $\rho$ as follows

$$
\begin{aligned}
S= & \frac{1}{2 \Lambda} \int d^{4} x \chi^{-1}[(1+\chi \operatorname{Tr} M) \\
& \left. \pm\left\{(1+\chi \operatorname{Tr} M)^{2}-2 \chi^{2}\left[\operatorname{Tr} M^{2}-\frac{1}{2}(\operatorname{Tr} M)^{2}\right]+8 \chi^{3}(\operatorname{det} M)\right\}^{1 / 2}\right]
\end{aligned}
$$

where $\chi:=(8 \rho)^{-1} \Lambda$. This is exactly the action found by Peldán in [6].

There are two distinct actions, depending on which square root is taken in (16). This is a consequence of the fact that (12) is non-linear in the unknown $X^{-1}$. Both roots are needed, since omitting a root would arbitrarily exclude some field configurations that satisfy the equations of motion?.

What if the same procedure used for the action (10) is applied to the equivalent action (8), that is without rewriting the constraint $\operatorname{Tr} X=\Lambda$ in the form (9)? It turns out that although the $\mathrm{X}$ equation of motion, $M=2 \rho X^{2}$, is simpler than (12), it is harder to solve for the invariant $\operatorname{Tr} X$ that appears in the Lagrangian. The independent traces of the equation $M=2 \rho X^{2}$ can be used to obtain a quartic

\footnotetext{
${ }^{2}$ Note that we disagree here with Peldán [6], who argues that one should keep only the root that agrees with the vacuum action (2) in the limit of a vanishing $\Lambda$.
} 
equation for $\operatorname{Tr} X$, but we have been unable to obtain an explicit expression for its solutions.

\section{ACKOWLEDGEMENTS}

We thank Abhijit Kshirsagar for a useful discussion. This work was supported by NSF grants PHY 8910226 and PHY 9112240. R.C. gratefully acknowledges support by a CONACyT post-doctoral fellowship, and by the Centro de Investigaciones y Estudios Avanzados, I.P.N. (Mexico). 


\section{References}

[1] Ashtekar A., Phys. Rev. Lett. 57, 2244 (1986) ; Phys. Rev D 36, 1587 (1987);

[2] Capovilla R., Dell J., and Jacobson T., Phys. Rev. Lett. 63, 2325 (1989);

[3] Capovilla R., Dell J., and Jacobson T., Class. Quant. Grav. 8, 59 (1991);

[4] Bengtsson I., and Peldán P., Phys. Lett. B 244, 261 (1990);

[5] Peldán P., Phys. Lett. B 248, 62 (1990);

[6] Peldán P., Class. Quant. Grav. 8, 1765 (1991);

[7] Penrose R., and Rindler W., Spinors and Spacetime, vol. 2, (Cambridge U. Press, 1986);

[8] Capovilla R., Dell J., and Jacobson T., Erratum to Class. Quant. Grav. 8, 59 (1991) (to be published);

[9] Dadhich N., Koshti S., and Kshirsager A., Class. Quant. Grav. 8, L61 (1991);

[10] Plebanski J.F., Journ. Math. Phys. 18, 2511 (1977);

[11] Mason L., and Frauendiener J., "The Sparling 3-Form, Ashtekar Variables, and Quasi-local Mass", in Twistors in Mathematics and Physics, eds. Baston R., and Bailey T., (Cambridge U. Press, 1990);

[12] Capovilla R., Dell J., Jacobson T., and Mason L., Class. Quant. Grav. 8, 41 (1991);

[13] Capovilla R., Nucl. Phys. B, 373, 233 (1992);

[14] Bengtsson I., and Peldán P., Int. J. Mod. Phys. A 7, 1287 (1992). 